\title{
«Wir sind eine Drehscheibe»
}

\section{Daniel Lüthi}

Freier Journalist und Fotograf, Medientrainer, Bern

Das Licht im Mammographie-Raum ist weich und zum Teil rosa. Das macht die grosse Maschine, die weibliche Brüste durchleuchtet und Krebs sichtbar macht, etwas weniger bedrohlich. Und die ganze angespannte Situation wohl auch. «Die kurze Zeit, bis auf dem Bildschirm das Bild erscheint, ist mit sehr viel Stress verbunden", sagt Rahel Kubik. Sie hat diesen Moment als Chefärztin schon sehr oft erlebt. Und als Frau auch schon.

\section{Im Dunkeln arbeiten}

«Es ist wie in Flugzeugen», erklärt Kubik. "Auch dort haben viele Leute Angst, deshalb sollen sie sich möglichst wohl und sicher fühlen, und deshalb ist das Ambiente wichtig.» Das rosa Licht in einer von Spitzentechnik dominierten Welt zeigt: Weiche Faktoren ha- ben sich in der Radiologie einen Platz erobert. So, wie sich auch die Radiologie in der Medizin einen Platz erobern musste. «Früher waren wir in den Untergrund verbannt», erinnert sich Rahel Kubik, «weil wir ja ohnehin meist im Dunkeln arbeiten. Aber auch deshalb, weil wir als Fachärzte oft gar nicht richtig ernst genommen wurden. Immer wieder haben uns Kolleginnen und Kollegen aus anderen Spezialgebieten abschätzig als 'Fotografen' bezeichnet.»

Eben: Weil die Radiologen Bilder machen, mit bildgebenden Verfahren arbeiten. Weil sie Abweichungen von der Normalität abbilden, verborgene Ursachen von Leiden sichtbar machen, aus dem Inneren eines Körpers auf einen Bildschirm holen. "Wir sind breit ausgebildet, aber immer mehr fokussiert sich jede und jeder von uns auf gewisse Organe», erläutert Rahel

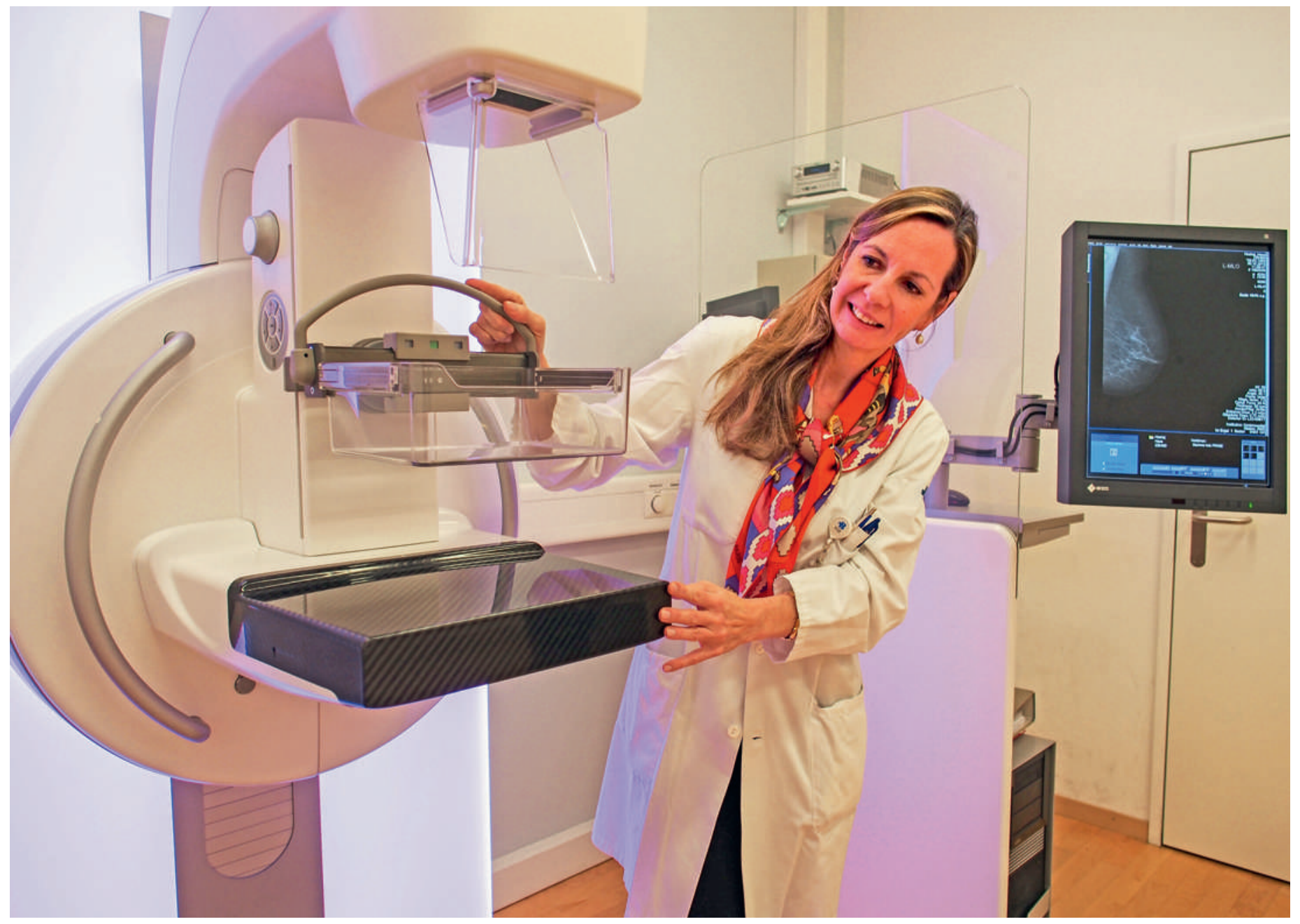


Kubik. So gibt es Kinder- und Neuro-Radiologen - oder eben Fachärztinnen wie sie, die auf "female imaging", also die Brüste und das Becken von Frauen spezialisiert sind. "Oft sind wir gefragt, wenn die Gynäkologen nicht mehr weiterkommen. Dann leisten wir bisweilen richtige Detektiv-Arbeit.»

Der Moment der Entdeckung, der Bruchteil einer Sekunde, wenn das Bild auf dem Bildschirm erscheint dieser Moment der Gewissheit kann grösstes Glück bedeuten: Eine gute Nachricht von einem Radiologen, eine Entwarnung ist ein Geschenk. Der Moment, der eine Vermutung zur Gewissheit macht, kann aber auch ein Leben völlig verändern. «Im schlechteren Fall sind wir die ersten, die einen Patienten mit der brutalen Realität konfrontieren müssen», sagt Kubik. Sie und ihre Kollegen bilden nicht nur ab, sondern sie beherrschen auch die Kunst, diese Bilder lesen und interpretieren zu können. Immer wieder sind diese diagnostischen Fähigkeiten gerade in einem Spital gefragt, werden die nächsten Schritte in der Radiologie entschieden: die richtigen Entscheide, Massnahmen, Therapien, Operationen. «Wir sind eine Drehscheibe», fasst Kubik zusammen.

\section{Immer mehr, immer schneller}

Im Büro der Chefärztin stehen vier Bildschirme. Drei davon sind speziell für die Diagnostik reserviert. Wir schauen uns ein paar Beispiele an. Ein Pankreas-MRI: 841 Bilder. Eine Leber: 1328 Bilder. Ein Thorax: 1443 Bilder. «Früher hatten wir bei einer Ganzkörper-Computertomographie sechs Filme à zwölf Bilder zu beurteilen», kommentiert Kubik, «heutzutage sind es bis 2000 Bilder. Es ist gewaltig, welche Informationsflut wir zu bewältigen haben. Und: Alles muss immer schneller gehen.»

Der Bruchteil einer Sekunde, wenn das Bild auf dem Bildschirm erscheint - dieser Moment kann grösstes Glück bedeuten.

Neben den Bildern des aktuellen Falls sind - wie bei einer Ampel mit Farben gekennzeichnet - auf einem der Computer-Bildschirme die Aufgaben aufgelistet, die heute noch zu erledigen sind: 7 Befunde stehen am Nachmittag an, 30 Befunde warten auf ihre Freigabe. «Bei 50 Aufgaben zeigt die Ampel rot an, dann habe ich Stress», sagt Kubik, «dann kommen die Telefone der Zuweiser.» Aber mit dem Diktieren der Befunde ist es ja nicht gemacht. Oft sind auch klärende Gespräche vonnöten.

Dazu kommen bei Rahel Kubik alle anderen Aufgaben, die sich bei ihr angesammelt haben: Als Chefärztin Radiologie ist sie für rund 110 Mitarbeitende verant-

\section{Rahel Kubik}

Prof. Dr. med. Rahel Kubik wurde 1967 in Marburg (Deutschland) geboren. Als Teenager kam sie zusammen mit ihren Eltern - beides Ärzte - nach Zürich, wo sie das Gymnasium beendete und Medizin studierte. Nach dem Staatsexamen 1991 arbeitete sie ein Jahr lang am Rockefeller University Hospital in New York, dann kam sie als Assistenzärztin ans USZ in Zürich, wo sie bis 2001 am Institut für Diagnostische Radiologie tätig war. 2000 bis 2004 absolvierte sie ein Nachdiplomstudium, das sie mit dem Master of Public Health abschloss. Seit 2002 arbeitet sie am Kantonsspital Baden, seit 2005 als Chefärztin Radiologie und Leiterin der Medizinischen Dienste. 2010 wurde sie zudem Mitglied der Geschäftsleitung. 2005 erhielt sie eine Titularprofessur an der Universität Zürich. Rahel Kubik ist verheiratet und Mutter einer zwölf- und einer achtjährigen Tochter. Sie lebt mit ihrer Familie in Zürich.

wortlich, davon sind etwa ein Drittel Ärztinnen und Ärzte. Als Leiterin des Departementes Medizinische Dienste ist sie Chefin von doppelt so vielen Personen. Ihr Hauptproblem beim Personal: «Wir leiden unter einem Mangel an Fachärzten. Das Ausland hat nicht geschlafen und bezahlt Fachärzte inzwischen auch fair - viele unserer Kolleginnen und Kollegen aus Deutschland beispielsweise gehen zurück. Ein eigentliches Nadelöhr sind die Weiterbildungsstätten.» Als Mitglied der Geschäftsleitung des Spitals ist Kubik an strategischen Entscheiden beteiligt. Und als Vorstandsmitglied ihrer Fachgesellschaft befasst sie sich unter anderem damit, das Image ihrer Zunft zu verbessern, Vorurteile zu revidieren. Zum Beispiel die Aussage, Radiologen seien vor allem Kostenverursacher.

\section{Technik kostet}

«Wir sind stark von der Technik abhängig», erklärt Rahel Kubik, «und Technik hat ihren Preis. Das macht uns immer wieder zum Angriffspunkt.» Kritisiert werden beispielsweise unnötige Zweitserien. «Ja, auch unter den Radiologen gibt es schwarze Schafe», stellt sie fest, "gerade private Institute stehen unter einem unternehmerischen Erfolgsdruck. Und es gibt in der Radiologie Investoren, die Rosinen picken und möglichst viel verdienen wollen, was tatsächlich irritierend ist.» Grundsätzlich sei festzuhalten: «Wir Radiologen machen à priori keine Selbst-Zuweisung, wir arbeiten nur auf Anweisung.» Sie verweist also auf die hohen Ansprüche der zuweisenden Ärzte: «Viele haben Angst, etwas zu verpassen, und wollen deshalb viele Bilder. Medico-legale Aspekte spielen dabei sicher eine Rolle. Heute werden massiv mehr Bilder angefordert als früher.» 
Und: Sie nimmt die Patientinnen und Patienten in die Pflicht: «Auch die Anspruchshaltung der Gesellschaft ist hoch. Heute will jeder mit 75 Jahren noch Golf spielen gehen. Jüngere Menschen stehen oft unter einem Beschleunigungs-Druck, abwarten hat da keinen Platz mehr, möglichst viel muss möglichst rasch klar sein.» Die andere Seite dieser Diskussion ist laut Kubik die, dass dank einer guten und schnellen Diagnose oft Kosten gespart werden können.

\section{Karriere und Familie}

Welches sind die "privaten Kosten» für eine Frau, die derart eingespannt ist, auf so vielen verschiedenen Ebenen verantwortungsvolle Arbeit leistet? Ein Burnout sei für viele, die ähnlich viel zu bewältigen hätten, sicher eine mögliche Gefahr, sagt Rahel Kubik. «Für mich selber ist es kein Thema. Allein die Abwechslung, die ich geniesse, ist eine gute Prävention.» Und die Familie? Hat sie noch Platz? «Meine Familie ist für mich das Wichtigste», stellt sie ohne kleinstes Zögern fest. Aber wie kann sie dem Anspruch, der sich hinter dieser Aussage verbirgt, gerecht werden?

Familie und Karriere unter einen Hut zu bringen, sei zu einem grossen Teil eine Frage der Organisation und Planung, ist Kubik überzeugt. Flexible Arbeitszeiten kämen dem entgegen. "Die Radiologie eignet sich besonders gut für Teilzeitarbeit.» In ihrer Position sei dies keine Option, aber ein Doppel-Einkommen erlaube es, bei der Kinderbetreuung oder im Haushalt Unterstützung zu beanspruchen. Seit Jahren schon hat die Familie eine englische Nanny beschäftigt, regelmässig kommen Mutter und Schwiegermutter zum Einsatz. «Ein gutes Backup gibt Freiheiten», sagt Kubik, und: «Das Haupt-Obstakel ist in der Schweiz das Schul- system. Fremdbetreuung oder -begleitung haben hierzulande keine Kultur.»

Zweierlei seien für sie besonders wichtig: «Ich habe den richtigen Partner. Und unkomplizierte, gesunde Kinder.» Wenn sie mit ihnen zusammen sei, versuche sie diese Zeit als 'Quality time' zu gestalten. Dann koche man zusammen oder treibe man gemeinsam Sport. Auch während der Arbeit habe sie für ihre Familie immer ein offenes Ohr. Wenn eine ihrer Töchter unbedingt mit ihr telefonieren wolle, verlasse sie dafür jederzeit auch wichtige Sitzungen. Ohne falsche Entschuldigung übrigens: «Ich sage dann jeweils ganz offen, meine Tochter wolle mit mir sprechen.»

Und wenn diese Radiologin zu Beginn eines neuen Jahres sich selber, ihre Hoffnungen und Befürchtungen ein wenig durchleuchtet, was wird da sicht- und spür-

\section{"Allein die Abwechslung, die ich geniesse, ist} eine gute Prävention.»

bar? Über ihren Beruf spricht Rahel Kubik lieber als über sich selbst. Und doch verlangsamt sie jetzt ihr Sprechtempo und erlaubt einen kleinen Einblick ins eigene Innere: "Ich wäre zufrieden, wenn es so weitergehen würde wie bisher», sagt sie. "Langsam aber sicher komme ich in ein Alter, wo das Eintretensrisiko von Krankheiten grösser wird. So gesehen hoffe ich, dass ich selber künftig möglichst wenig Radiologie brauchen werde.»

\section{Die nächste Begegnung mit ...}

Am Ende jeden Monats stellt die Schweizerische Ärztezeitung eine Persönlichkeit vor, die sich im Gesundheitswesen engagiert. Im Februar schildert Daniel Lüthi seine Begegnung mit Urs Daendliker, Physiotherapeut in Sion. 